\title{
Absorción de nitrógeno durante el desarrollo del cultivo de cebada maltera (Hordeum vulgare L.) Variedad metcalfe. Imbabura
}

Nitrogen absorption during the development of the cultivation of malley barley (Hordeum vulgare L.). Variety metcalfe. Imbabura

Absorção de nitrogênio durante o desenvolvimento do cultivo de cevada (Hordeum vulgare L.) Variedade metcalfe. Imbabura

Daniela Albán M. dalban_m@outlook.es

Marcelo Calvache U. mcalvache20@gmail.com

Javier Garófalo S.

jaalgaso@yahoo.com

\section{Universidad Central del Ecuador - Instituto Nacional de Investigaciones Agropecuarias, Ecuador}

Artículo recibido noviembre 2017, arbitrado mayo 2018 y publicado en septiembre 2018

\section{RESUMEN}

La investigación planteó desarrollar una tecnología adecuada para el manejo de la fertilización nitrogenada complementaria en cebada cervecera mediante un estudio de absorción de nitrógeno. Esta investigación se desarrolló en la provincia de Imbabura, Los métodos usados fueron los experimentos se establecieron en la Hcda. Cobuendo (2340 msnm) ubicada en el cantón Antonio Ante y en la Hcda. Pisangacho (2700 msnm) situada en el cantón Urcuquí. Como resultado se obtuvo que el estudio de absorción de nitrógeno realizado en la localidad de Cobuendo, permitió mostrar que la acumulación de nitrógeno en la biomasa aérea total en función del tiempo, presentó una tendencia sigmoidea típica. La acumulación de nitrógeno en la biomasa aérea total se ajustó a una regresión logística normal.

Palabras clave: absorción de nitrógeno; cultivo; cebada malteada

\begin{abstract}
The research aimed to develop a suitable technology for the management of complementary nitrogen fertilization in beer barley through a nitrogen absorption study. This investigation was developed in the province of Imbabura. The methods used were the experiments established in the Hcda. Cobuendo (2340 masl) located in the Antonio Ante canton and in the Hcda. Pisangacho (2700 masl) located in the Urcuquí canton. As a result, it was obtained that the nitrogen absorption study carried out in the Cobuendo locality showed that the accumulation of nitrogen in the total aerial biomass as a function of time presented a typical sigmoid trend. Nitrogen accumulation in total aboveground biomass was fitted to a normal logistic regression.
\end{abstract}

Key words: nitrogen absorption; culture; malted barley 
RESUMO

A pesquisa teve como objetivo desenvolver uma tecnologia adequada para o manejo da adubação nitrogenada complementar na cevada de cerveja através de um estudo de absorção de nitrogênio. Esta investigação foi desenvolvida na província de Imbabura e os métodos utilizados foram os experimentos estabelecidos no Hcda. Cobuendo (2340 msnm) localizado no cantão Antonio Ante e no Hcda. Pisangacho (2700 msnm) localizado no cantão Urcuquí. Como resultado, foi obtido que o estudo de absorção de nitrogênio realizado na localidade de Cobuendo mostrou que o acúmulo de nitrogênio na biomassa aérea total em função do tempo apresentava uma tendência sigmóide típica. 0 acúmulo de nitrogênio na biomassa total acima do solo foi ajustado a uma regressão logística normal.

Palavras-chave: absorção de nitrogênio; cultivo; cevada maltada

\section{INTRODUCCIÓN}

La cebada (Hordeum vulgare L.) es una de las gramíneas más importantes a nivel mundial, ocupa el quinto lugar entre los cereales de mayor producción, (FAOSTAT, 2012). Este cultivo se destina, principalmente, para la alimentación humana, consumo animal y para uso industrial en la producción de malta, elaboración de cerveza y otras bebidas destiladas, (INEC, 2010). En Ecuador la demanda de cebada para consumo humano, es casi satisfecha con la producción local. Sin embargo, la industria cervecera importa entre 35000 - $40000 \mathrm{t}$ anuales de cebada para procesamiento industrial, (Banco Central del Ecuador - SINAGAP, 2014).

En el año 2009, el Programa de Cereales del INIAP en convenio con Cervecería Nacional, seleccionó a la variedad Metcalfe, como resultado de un estudio de adaptación de variedades de cebada cervecera, (INIAP, 2010). La identificación de una variedad no garantiza una producción que cumpla con los requisitos exigidos por la industria. Una de las características más importantes es el contenido de proteína en el grano que debe ser del 10 al 12\%, (Giménez et al., 2007; Lazzari et al., 2001; Arias, 1996). Por esta razón, en las variedades cerveceras se debe prestar mayor atención a la fertilización nitrogenada que es el factor determinante para asegurar altos rendimientos, buena calidad maltera y bajos costos de producción, (Lazzari et al., 2001).

En esta investigación se planteó desarrollar una tecnología adecuada para el manejo de la fertilización nitrogenada complementaria en cebada cervecera mediante un estudio de absorción de nitrógeno. La información generada permite conocer la etapa de mayor asimilación del nutriente, asegurando que la cantidad de nitrógeno que el cultivo puede aprovechar sea mayor y al mismo tiempo minimizando el riesgo de afectar negativamente al ambiente, (Castro, 2004; Henríquez y otros 2005). Considerando estos antecedentes se plantearon los siguientes objetivos: Establecer la dosis óptima de nitrógeno complementario y la época de aplicación más adecuada para conseguir el mejor rendimiento y contenido de proteína del grano de cebada. Determinar la absorción de nitrógeno del cultivo de cebada cervecera.

\section{MATERIALES Y MÉTODOS}

Esta investigación se desarrolló en la provincia de Imbabura. Los experimentos se establecieron en la Hcda. Cobuendo (2340 msnm) ubicada en el cantón Antonio Ante y en la Hcda. Pisangacho (2700 msnm) situada en el cantón Urcuquí. Las localidades se encuentran en una región climática Seco Temperada con una temperatura promedio anual de $15.8{ }^{\circ} \mathrm{C}$, una precipitación acumulada anual $620.9 \mathrm{~mm}$ y una humedad relativa promedio anual de $82 \%$, (INAMHI, 2010). 
Los factores en estudio fueron: épocas de aplicación y dosis de nitrógeno complementario. Las etapas fenológicas de aplicación del fertilizante nitrogenado fueron macollamiento, Zadoks 22 (Z22) y producción de nudos, Zadoks 30 (Z30), se evaluaron niveles crecientes de nitrógeno complementario $(0,25,50,75,100$ y $125 \mathrm{~kg}$ $\mathrm{N} / \mathrm{ha}$ ). En el experimento se utilizó un Diseño de Parcela Dividida con tres repeticiones. La unidad experimental fue de $7 \times 3 \mathrm{~m}=21 \mathrm{~m}^{2}$.

La preparación del suelo consistió en una labor de arada y dos pases de rastra. Antes de siembra se tomó una muestra compuesta de suelo para el análisis de laboratorio. La siembra se realizó de forma manual al voleo a una densidad de $135 \mathrm{~kg} / \mathrm{ha}$. En la siembra se aplicó todo el fósforo, potasio, azufre y magnesio. La cantidad de nitrógeno aplicado en la siembra fue $25 \mathrm{~kg} / \mathrm{ha}$. Posteriormente se realizó aplicaciones de fertilizante nitrogenado complementario en las etapas fenológicas Z22 y Z30. También se realizó el control de enfermedades y malezas a los $30 \mathrm{y}$ 45 días después de la siembra (dds), respectivamente.
En las dos localidades al momento de la cosecha, las variables evaluadas fueron: altura de plantas, rendimiento, componentes de rendimiento (número de granos por espiga, número de espigas por planta y peso de mil granos), peso hectolítrico y porcentaje de proteína. Adicionalmente en Cobuendo se realizó cuatro muestreos de la biomasa aérea total durante el ciclo del cultivo (macollamiento, producción de nudos, espigamiento y madurez fisiológica), se determinó PMF, PMS, extracción de nitrógeno y el índice de cosecha. Complementariamente se realizó el análisis financiero.

\section{RESULTADOS Y DISCUSIÓN}

\section{Número de granos por espiga}

El análisis de varianza para número de granos por espiga, tabla 1, identifica diferencias significativas para niveles de Nitrógeno en la localidad de Cobuendo, mientras que, para Pisangacho el análisis muestra diferencias altamente significativas. El efecto lineal presenta alta significación en las dos localidades. 
Tabla 1. Análisis de varianza para dos variables en la evaluación de dosis y épocas de aplicación de nitrógeno complementario en cebada maltera (Hordeum vulgare L.). Imbabura.

\begin{tabular}{|c|c|c|c|c|c|c|}
\hline \multirow[t]{3}{*}{ F. de V. } & \multirow[t]{3}{*}{ G.L } & & \multicolumn{4}{|c|}{ CUADRADOS MEDIOS } \\
\hline & & & \multicolumn{2}{|c|}{ Granos/espiga } & \multicolumn{2}{|c|}{ Espigas/planta } \\
\hline & & & $\mathbf{C}$ & $\mathbf{P}$ & C & $\mathbf{P}$ \\
\hline TOTAL & 35 & & & & & \\
\hline REPETICIONES & 22 & & 2.42 n.s & 0.04 n.s & 11.52 n.s & 6.69 n.s \\
\hline ÉPOCAS (E ) & 1 & & 3.06 n.s & 0.04 n.s. & 1.48 n.s & 2.51 n.s \\
\hline ERROR (a) & 5 & & 9.94 & 0.40 & 7.79 & 3.84 \\
\hline NITRÓGENO (N) & & & 15.27 & $5.78 * *$ & $12.05^{* *}$ & $11.52 * *$ \\
\hline Lineal & & 1 & $65.06 * *$ & $24.98 * *$ & $54.36 * *$ & $54.94 * *$ \\
\hline Cuadrático & & 1 & 3.63 n.s & $2.75 *$ & 1.34 n.s. & 0.03 n.s \\
\hline Cúbico & & 1 & 6.88 n.s. & 0.15 n.s. & 4.46 n.s & 2.04 n.s \\
\hline Resto & & & 0.40 n.s & 0.50 n.s. & 0.04 n.s & 0.29 n.s \\
\hline ExN & 5 & 2 & 4.19 n.s. & 0.20 n.s. & 2.76 n.s & $1.10 \mathrm{n} . \mathrm{s}$ \\
\hline ERROR (b) & 20 & & 4.70 & 0.45 & 2.22 & 1.15 \\
\hline Promedio & & & 23.33 & 26.36 & 8.55 & 7.13 \\
\hline C.V. (a) \% & & & 13.50 & 2.39 & 32.63 & 27.49 \\
\hline C.V. (b) \% & & & 9.30 & 2.54 & 17.43 & 15.01 \\
\hline
\end{tabular}

C: Cobuendo P: Pisangacho

En ambas localidades la prueba de Tukey $(\alpha=0.05)$ para Nitrógeno, Tabla 2, establece que la dosis con el número de granos por espiga promedio más destacado fue $100 \mathrm{~kg}$ $\mathrm{N} / \mathrm{ha}$, obteniendo un valor promedio para Cobuendo de 25.37 granos/espiga y para Pisangacho el valor obtenido fue de 27.52 granos/espiga. Además, en las dos localidades se observó una tendencia lineal positiva para nitrógeno hasta la aplicación del nivel de 100 $\mathrm{kg} \mathrm{N} / \mathrm{ha}$; es decir que, al incrementar las dosis de nitrógeno incrementó el número de granos por espiga.

De acuerdo al análisis de varianza para número de espigas por planta, Tabla 1 , en la localidad de Cobuendo y Pisangacho, se detectó diferencias altamente significativas para niveles de Nitrógeno y para el efecto lineal de este factor.

La prueba de Tukey $(\alpha=0.05)$ para Nitrógeno, Tabla 2, establece que la dosis más destacada para Cobuendo fue $100 \mathrm{~kg} \mathrm{~N} / \mathrm{ha}$ con 10.07 espigas/planta; mientras que, para Pisangacho fue la dosis de $125 \mathrm{~kg} \mathrm{~N} /$ ha con un valor de 9.20 espigas/planta. Además, en las dos localidades se observó una tendencia lineal positiva para nitrógeno; es decir que, al incrementar las dosis de nitrógeno incrementó el número de espigas por planta. 
Tabla 2. Promedios y prueba de significación para dos variables en la evaluación de dosis y épocas de aplicación de nitrógeno complementario en cebada maltera (Hordeum vulgare L.). Imbabura.

\begin{tabular}{lcllc}
\hline & \multicolumn{2}{c}{ Promedios } & \multicolumn{2}{c}{ Factores } \\
\hline & $\begin{array}{c}\text { Granos/espiga } \\
\text { C }\end{array}$ & P & C & P \\
Épocas (E) & & & & \\
e1 (Z22) & 23.04 & 26.40 & 8.34 & 6.86 \\
e2 (Z22 + Z30) & 23.62 & 26.33 & 8.75 & 7.39 \\
Nitrógeno (N) & & $\mathbf{1}$ & $\mathbf{1}$ & $\mathbf{1}$ \\
n0 (0 kg N/ha) & $21.32 \mathrm{~b}$ & $24.79 \mathrm{c}$ & $6.82 \mathrm{~b}$ & $5.18 \mathrm{c}$ \\
n1 (25 kg N/ha) & $21.70 \mathrm{ab}$ & $25.74 \mathrm{bc}$ & $7.07 \mathrm{~b}$ & $6.17 \mathrm{bc}$ \\
n2 (50 kg N/ha) & $23.07 \mathrm{ab}$ & $26.38 \mathrm{ab}$ & $8.10 \mathrm{ab}$ & $7.13 \mathrm{bc}$ \\
n3 (75 kg N/ha) & $24.12 \mathrm{ab}$ & $26.68 \mathrm{ab}$ & $9.45 \mathrm{ab}$ & $7.17 \mathrm{~b}$ \\
n4 (100 kg N/ha) & $25.37 \mathrm{a}$ & $27.52 \mathrm{a}$ & $10.07 \mathrm{a}$ & $7.90 \mathrm{ab}$ \\
n5 (125 kg N/ha) & $24.42 \mathrm{ab}$ & $27.07 \mathrm{a}$ & $9.78 \mathrm{a}$ & $9.20 \mathrm{a}$ \\
Interacción ExN & & & & \\
e1n0 & 20.10 & 24.71 & 6.33 & 4.90 \\
e1n1 & 21.17 & 25.71 & 6.70 & 6.03 \\
e1n2 & 22.30 & 26.55 & 7.73 & 7.10 \\
e1n3 & 23.43 & 26.43 & 8.33 & 6.13 \\
e1n4 & 25.83 & 27.76 & 10.33 & 8.13 \\
e1n5 & 25.40 & 27.22 & 10.63 & 8.87 \\
e2n0 & 22.53 & 24.86 & 7.30 & 5.47 \\
e2n1 & 22.23 & 25.77 & 7.43 & 6.30 \\
e2n2 & 23.83 & 26.22 & 8.47 & 7.17 \\
e2n3 26.92 & 10.57 & 8.20 \\
e2n4 & 24.80 & 26.92 & 9.80 & 7.67 \\
e2n5 & 24.90 & 27.27 & & \\
\hline
\end{tabular}

1: Prueba de Tukey al 5\%; C: Cobuendo, P: Pisangacho

\section{Rendimiento}

El análisis de varianza para rendimiento en las localidades de Cobuendo y Pisangacho, Tabla 3, detectó diferencias altamente significativas para niveles de Nitrógeno y para el polinomio lineal.

La prueba de Tukey $(\alpha=0.05)$ para Nitrógeno, Tabla 4, establece que la dosis con el rendimiento promedio más destacado para Cobuendo fue $125 \mathrm{~kg} \mathrm{~N} / \mathrm{ha}$ con $4.51 \mathrm{t} / \mathrm{ha}$; mientras que, para Pisangacho fue la dosis de $100 \mathrm{~kg} \mathrm{~N} / \mathrm{ha}$ con un valor de $4.11 \mathrm{t} / \mathrm{ha}$.

La respuesta de rendimiento a los niveles de nitrógeno complementario, muestran un incremento lineal en la localidad de
Cobuendo; es decir que, al aumentar las dosis de nitrógeno existe un incremento en el rendimiento. En Pisangacho se observa que el nitrógeno incrementó el rendimiento hasta la aplicación del nivel de $100 \mathrm{~kg} \mathrm{~N} / \mathrm{ha}$; sin embargo, con el nivel más alto n5 $(125 \mathrm{~kg}$ $\mathrm{N} / \mathrm{ha}$ ) se observa una ligera disminución en el rendimiento ( $3.72 \mathrm{t} / \mathrm{ha}$ ), esto posiblemente se debe a un desequilibrio entre nutrimentos, provocado por el aumento excesivo en la concentración de $\mathrm{N}$ de la planta, (Bertsch, 1998). En general, este comportamiento también se observó en los componentes de rendimiento. 
Tabla 3. Análisis de varianza para dos variables en la evaluación de dosis y épocas de aplicación de nitrógeno complementario en cebada maltera (Hordeum vulgare L.). Imbabura. 2010.

\begin{tabular}{|c|c|c|c|c|c|c|}
\hline \multirow{3}{*}{$\begin{array}{l}\text { F. de V. } \\
\text { TOTAL }\end{array}$} & \multirow{3}{*}{$\begin{array}{l}\text { G.L } \\
35\end{array}$} & \multicolumn{5}{|c|}{ CUADRADOS MEDIOS } \\
\hline & & & Rendimi & & Proteína & \\
\hline & & & C & $\mathbf{P}$ & C & $\mathbf{P}$ \\
\hline REPETICIONES & 2 & & $2.60 * *$ & $0.96 \mathrm{n} . \mathrm{s}$ & $1.33 \mathrm{n} . \mathrm{s}$ & $4.32 \mathrm{n} . \mathrm{s}$ \\
\hline ÉPOCAS (E) & 1 & & 0.03 n.s & 0.01 n.s. & 0.19 n.s & 0.70 n.s \\
\hline ERROR (a) & 2 & & 0.48 & 0.49 & 0.57 & 1.58 \\
\hline $\begin{array}{l}\text { NITRÓGENO } \\
\text { (N) }\end{array}$ & 5 & & $1.44^{* *}$ & $1.89 * *$ & $5.57 * *$ & $17.22 * *$ \\
\hline Lineal & & 1 & $6.46^{* *}$ & $7.37^{* *}$ & $24.64 * *$ & $80.99 * *$ \\
\hline Cuadrático & & 1 & 0.72 n.s & $0.96 \mathrm{n} . \mathrm{s}$ & 0.15 n.s & 1.15 n.s \\
\hline Cúbico & & 1 & $0.02 \mathrm{n} . \mathrm{s}$ & $0.84 \mathrm{n} . \mathrm{s}$ & 2.66 n.s & $1.23 \mathrm{n} . \mathrm{s}$ \\
\hline Resto & & 2 & 0.02 n.s. & $0.14 \mathrm{n} . \mathrm{s}$ & 0.20 n.s & $1.32 \mathrm{n} . \mathrm{s}$ \\
\hline ExN & & & 0.21 n.s. & $0.11 \mathrm{n} . \mathrm{s}$ & 0.55 n.s. & $1.64 \mathrm{n} . \mathrm{s}$ \\
\hline ERROR (b) & 5 & & 0.12 & 0.27 & 0.73 & 1.48 \\
\hline Promedio & 20 & & $4.06 \mathrm{t} / \mathrm{ha}$ & $3.44 \mathrm{t} / \mathrm{ha}$ & $12.81 \%$ & $11.78 \%$ \\
\hline C.V. (a) \% & & & 17.00 & 20.35 & 5.85 & 10.70 \\
\hline C.V. (b) \% & & & 8.62 & 15.12 & 6.64 & 10.36 \\
\hline
\end{tabular}

C: Cobuendo

P: Pisangacho

\section{Porcentaje de Proteína}

El análisis de varianza para porcentaje de proteína, Tabla 4, en la localidad de Cobuendo y Pisangacho, identifica diferencias altamente significativas para niveles de Nitrógeno y para el polinomio lineal.

En ambas localidades la prueba de Tukey $(\alpha=0.05)$ para Nitrógeno, Tabla 4 , establece que la dosis de $100 \mathrm{~kg} \mathrm{~N} /$ ha se destaca con un mayor porcentaje de proteína, obteniendo un valor promedio para Cobuendo de $14.01 \%$ y para Pisangacho el valor obtenido fue $13.68 \%$.

Tomando en cuenta los requerimientos de la industria, en la localidad de Cobuendo los niveles de proteína que se encuentran entre el 10 y $12 \%$, se obtuvieron con las dosis de 25 y $50 \mathrm{~kg} \mathrm{~N} / \mathrm{ha}$; mientras que, en Pisangancho fueron las dosis de 25, 50 y $75 \mathrm{~kg}$ N/ha. 
Tabla 4. Promedios y prueba de significación para dos variables en la evaluación de dosis y épocas de aplicación de nitrógeno complementario en cebada maltera (Hordeum vulgare L.). Imbabura.

\begin{tabular}{|c|c|c|c|c|}
\hline \multicolumn{2}{|c|}{ Factores } & \multicolumn{3}{|c|}{ Promedios } \\
\hline & \multicolumn{2}{|c|}{ Rendimiento (t/ha) } & \multicolumn{2}{|c|}{ \% de Proteína } \\
\hline & C & $\mathbf{P}$ & C & $\mathbf{P}$ \\
\hline \multicolumn{5}{|l|}{ Épocas (E } \\
\hline e1 (Z22) & 4.04 & 3.42 & 12.88 & 11.64 \\
\hline e2 (Z22 + Z30) & 4.09 & 3.46 & 12.73 & 11.92 \\
\hline Nitrógeno $(\mathrm{N})$ & 1 & 1 & 1 & 1 \\
\hline n0 (0 kg N/ha) & $3.23 \mathrm{c}$ & $2.66 \mathrm{c}$ & $11.88 \mathrm{~b}$ & $9.38 \mathrm{c}$ \\
\hline n1 (25 kg N/ha) & $3.75 \mathrm{bc}$ & $3.02 \mathrm{bc}$ & $11.87 \mathrm{~b}$ & $10.69 \mathrm{bc}$ \\
\hline n2 (50 kg N/ha) & $4.15 \mathrm{ab}$ & $3.22 \mathrm{abc}$ & $12.19 \mathrm{~b}$ & $11.04 \mathrm{bc}$ \\
\hline n3 (75 kg N/ha) & $4.27 \mathrm{ab}$ & $3.89 \mathrm{ab}$ & $13.09 \mathrm{ab}$ & $12.42 \mathrm{ab}$ \\
\hline n4 (100 kg N/ha) & $4.47 \mathrm{a}$ & $4.11 \mathrm{a}$ & $14.01 \mathrm{a}$ & 13.68 a \\
\hline n5 (125 kg N/ha) & $4.51 \mathrm{a}$ & $3.72 \mathrm{ab}$ & $13.81 \mathrm{a}$ & $13.46 \mathrm{a}$ \\
\hline \multicolumn{5}{|l|}{ Interacción ExN } \\
\hline e1n0 & 3.30 & 2.77 & 12.36 & 9.45 \\
\hline e1n1 & 3.93 & 2.90 & 11.68 & 9.85 \\
\hline e1n2 & 3.83 & 3.40 & 11.89 & 10.98 \\
\hline e1n3 & 4.08 & 3.84 & 13.02 & 11.80 \\
\hline e1n4 & 4.55 & 4.05 & 14.30 & 14.30 \\
\hline e1n5 & 4.53 & 3.55 & 14.03 & 13.45 \\
\hline $\mathrm{e} 2 \mathrm{n} 0$ & 3.17 & 2.54 & 11.41 & 9.31 \\
\hline e2n1 & 3.57 & 3.14 & 12.05 & 11.53 \\
\hline $\mathrm{e} 2 \mathrm{n} 2$ & 4.46 & 3.04 & 12.50 & 11.10 \\
\hline e2n3 & 4.46 & 3.94 & 13.16 & 13.04 \\
\hline $\mathrm{e} 2 \mathrm{n} 4$ & 4.40 & 4.17 & 13.71 & 13.06 \\
\hline $\mathrm{e} 2 \mathrm{n} 5$ & 4.49 & 3.90 & 13.58 & 13.47 \\
\hline
\end{tabular}

\section{Nitrógeno Extraído}

En la localidad de Cobuendo, el análisis de varianza para nitrógeno extraído, Tabla 5, detectó diferencias altamente significativas para la biomasa aérea total y grano. En los dos casos la prueba de polinomios ortogonales presenta una tendencia lineal con alta significación. La interacción épocas por niveles de nitrógeno presentó diferencias significativas para la biomasa aérea total.
La prueba de Tukey $(\alpha=0.05)$ para Nitrógeno, Tabla 6, establece que la dosis con la extracción promedio más destacada para la biomasa aérea total fue $125 \mathrm{~kg} \mathrm{~N} /$ ha con $273.72 \mathrm{~kg} \mathrm{~N} / \mathrm{ha}$; mientras que, para el grano el valor promedio de nitrógeno extraído más destacado se obtuvo con la dosis de $100 \mathrm{~kg}$ $\mathrm{N} / \mathrm{ha}$ con $186.23 \mathrm{~kg} \mathrm{~N} / \mathrm{ha}$. 
Tabla 5. Análisis de varianza para nitrógeno extraído en la evaluación de dosis y épocas de aplicación de nitrógeno complementario en cebada maltera (Hordeum vulgare L.). Cobuendo, Imbabura.

\begin{tabular}{lllll}
\hline F. de V. & G.L. & \multicolumn{2}{c}{ CUADRADOS MEDIOS } \\
\hline & & total & Biomasa aérea & Grano \\
TOTAL & 35 & & & \\
REPETICIONES & 2 & & 370.21 n.s. & $305.26 \mathrm{n} . \mathrm{s}$ \\
ÉPOCAS (E) & 1 & & 169.39 n.s. & $933.96 \mathrm{n} . \mathrm{s}$ \\
ERROR (a) & 2 & & 1726.76 & 1814.46 \\
$\quad$ NITRÓGENO (N) & 5 & & $14897.53^{* *}$ & $6159.36^{* *}$ \\
Lineal & & 1 & $69732.85^{* *}$ & $28206.53^{* *}$ \\
Cuadrático & & 1 & $3574.35 \mathrm{n} . \mathrm{s}$. & $946.83 \mathrm{n} . \mathrm{s}$ \\
Cúbico & & 1 & $65.94 \mathrm{n} . \mathrm{s}$ & $14.99 \mathrm{n} . \mathrm{s}$ \\
Resto & 5 & & $555.78 \mathrm{n} . \mathrm{s}$ & $814.5 \mathrm{n} . \mathrm{s}$ \\
ExN & 20 & & $2544.79^{*}$ & $924.64 \mathrm{n} . \mathrm{s}$ \\
ERROR (b) & & & 910.17 & 453.63 \\
Promediokg N/ha & & & 18.81 & 149.09 \\
C.V. (a) \% & & & 13.48 & 28.57 \\
C.V. (b) \% & & & & 14.29 \\
\hline
\end{tabular}

Prueba de Tukey al 5\%; C: Cobuendo, P: Pisangacho

En general, se observa una tendencia lineal positiva para el factor nitrógeno en la biomasa aérea total; es decir que, al aumentar las dosis de nitrógeno existe un incremento en la extracción de este elemento, siendo la época 2 la que mayor extracción posee, corroborando que las aplicaciones fraccionadas hacen más eficiente la utilización de N, (García, 2009), induciendo a una mejor asimilación de este nutriente.

La tendencia del nitrógeno acumulado en la biomasa aérea total durante el ciclo del cultivo (macollamiento- 20dds, producción de nudos-44dds, espigamiento-75dds y madurez fisiológica-105 dds), se ajustó a una regresión logística normal, (Gráfico 1). Se puede apreciar mínimas diferencias en la acumulación de nitrógeno a los 20 dds y el nitrógeno aumenta lentamente hasta los 44 dds (fase exponencial). Luego se produce un incremento importante en la extracción de nitrógeno desde los 44 dds hasta los 105 dds (fase lineal y fase de senescencia), (Salisbury y Ross, 2000), mostrando el efecto del nitrógeno aplicado en la etapa de macollamiento (Z22) y producción de nudos (Z30).

Al momento de la cosecha, la curva de acumulación de nitrógeno en la biomasa aérea total estimó una extracción de nitrógeno de $223.15 \mathrm{~kg} \mathrm{~N} /$ ha con la época $1 \mathrm{y}$ $236.69 \mathrm{~kg} \mathrm{~N} / \mathrm{ha}$ con la época 2 , lo que indica una mejor asimilación de este nutriente en la época 2, (Gráfico 1). 
Tabla 6. Promedios y prueba de significación para nitrógeno extraído en la evaluación de dosis y épocas de aplicación de nitrógeno complementario en cebada maltera (Hordeum vulgare L.). Cobuendo, Imbabura.

\begin{tabular}{lll}
\hline & Valores promedios (kg N/ha) & \\
\hline Factores & Biomasa aérea total & Grano \\
Épocas (E) & & \\
e1 (Z22) & 221.64 & 144.00 \\
e2 (Z22 + Z30) & 225.97 & 154.18 \\
Nitrógeno (N) & $\mathbf{1}$ & $\mathbf{1}$ \\
n0 (0 kg N/ha) & $143.60 \mathrm{c}$ & $99.11 \mathrm{~d}$ \\
n1 (25 kg N/ha) & $192.00 \mathrm{bc}$ & $132.75 \mathrm{~cd}$ \\
n2 (50 kg N/ha) & $224.94 \mathrm{ab}$ & $142.55 \mathrm{bc}$ \\
n3 (75 kg N/ha) & $236.66 \mathrm{ab}$ & $154.56 \mathrm{abc}$ \\
n4 (100 kg N/ha) & 271.89 & $186.23 \mathrm{a}$ \\
n5 (125 kg N/ha) & $273.72 \mathrm{a}$ & $179.35 \mathrm{ab}$ \\
Interacción ExN & $\mathbf{1}$ & \\
e1n0 & $131.44 \mathrm{~d}$ & 90.44 \\
e1n1 & $168.53 \mathrm{bcd}$ & 119.09 \\
e1n2 & $236.32 \mathrm{abc}$ & 140.81 \\
e1n3 & $216.47 \mathrm{abcd}$ & 138.27 \\
e1n4 & $273.16 \mathrm{a}$ & 177.95 \\
e1n5 & $303.90 \mathrm{a}$ & 197.44 \\
e2n0 & $155.77 \mathrm{~cd}$ & 107.78 \\
e2n1 & $215.48 \mathrm{abcd}$ & 146.41 \\
e2n2 & $213.57 \mathrm{abcd}$ & 144.29 \\
e2n3 & $256.86 \mathrm{ab}$ & 170.85 \\
e2n4 & $270.62 \mathrm{a}$ & 194.51 \\
e2n5 & $243.54 \mathrm{abc}$ & 161.26 \\
Prueba de Tukey al 5\% & & \\
\hline & & \\
\hline
\end{tabular}

$$
\begin{gathered}
\ddot{y}=\frac{283.93}{1+18.16^{*} \mathrm{e}^{(-0.04 * x)}} \\
\mathrm{R}^{2}=0.87
\end{gathered}
$$

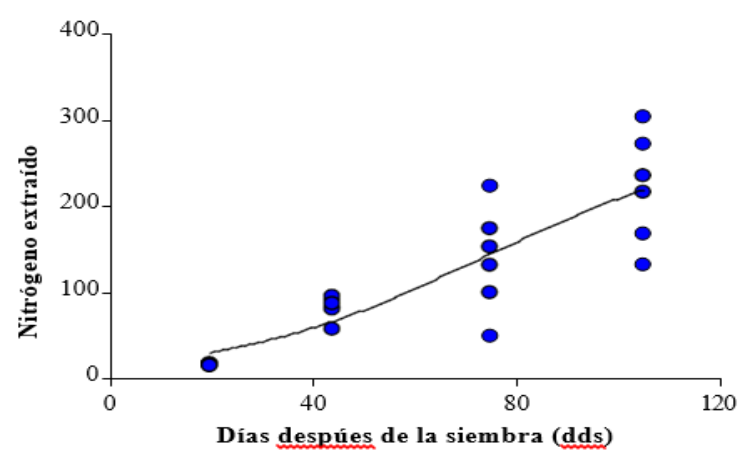

a) Época 1

$$
\begin{gathered}
\grave{y}=\frac{247.41}{1+29.09^{*} \mathrm{e}^{(-0.04 * \mathrm{x})}} \\
\mathrm{R}^{2}=0.95
\end{gathered}
$$

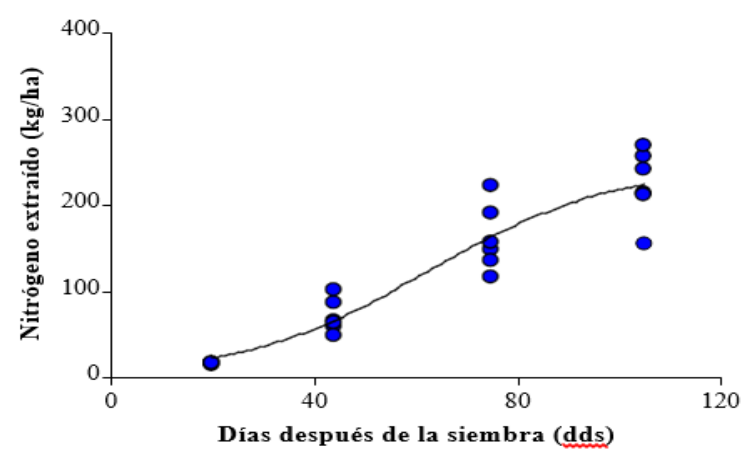

b) Época 2

Gráfico 1. Efecto de los niveles de nitrógeno en la acumulación del nitrógeno extraído de la biomasa aérea total, en dos épocas de aplicación (a y b). Cobuendo, Imbabura. 2010. 


\section{CONCLUSIONES}

En las localidades de Cobuendo y Pisangacho el cultivo de cebada maltera (Hordeum vulgare L.) variedad Metcalfe, respondió positivamente a la fertilización nitrogenada complementaria, los mejores rendimientos se consiguieron con la aplicación de $100 \mathrm{~kg} \mathrm{~N} / \mathrm{ha}$.

Las dosis de nitrógeno complementario de 25 y $50 \mathrm{~kg} \mathrm{~N} / \mathrm{ha}$ proporcionaron niveles de proteína requeridos por la industria en la localidad de Cobuendo; mientras que, en Pisangacho, se consiguió un contenido de proteína en el grano entre el 10 y $12 \%$ con las dosis de 25, 50 y $75 \mathrm{~kg} \mathrm{~N} / \mathrm{ha}$.

El estudio de absorción de nitrógeno realizado en la localidad de Cobuendo, permitió mostrar que la acumulación de nitrógeno en la biomasa aérea total en función del tiempo, presentó una tendencia sigmoidea típica. La acumulación de nitrógeno en la biomasa aérea total se ajustó a una regresión logística normal

$(\hat{y}=-a)$. La $_{(c * x)}$ absorción de nitrógeno en la biomasa aérea

Total fue $223.81 \mathrm{~kg} \mathrm{~N} /$ ha y la cantidad de

\section{$1+\mathrm{b} * e$}

Nitrógeno extraído en el grano fue $149.09 \mathrm{~kg} \mathrm{~N} / \mathrm{ha}$.

Aplicar la dosis de $25 \mathrm{~kg} \mathrm{~N} / \mathrm{ha}$ en la localidad de Cobuendo; mientras que, para Pisangacho se sugiere aplicar la dosis de 75 $\mathrm{kg}$ N/ha. Los niveles de nitrógeno complementario recomendados pueden ser aplicados en lugares que presenten características similares a los suelos de las localidades mencionadas.

\section{REFERENCIAS}

Arias, G. (1996). La calidad industrial de la cebada cervecera. In: Primera Reunión Latinoamericana de Cebada Cervecera.
Cochabamba, 1994. La Paz, BO. FAO. p. 141-183

Bertsch, GF, Hencken, K. y Esbensen, H. (1998). Desintegración nuclear de los núcleos borromeanos. Physical Review C 57 (3), 1366

BCE (Banco Central del Ecuador) - SINAGAP (Sistema de Información Nacional de Agricultura, Ganadería, Acuacultura y Pesca). (2014). Comercio Exterior Importaciones y Exportaciones BCE. Quito, EC. Consultado 20 sep. 2014. Disponible en: http://sinagap.agricultura.gob.ec/import aciones-y- exportaciones-bce

Castro, R.; GALVIS, A. (2004). Demanda de nitrógeno en tomate de cáscara. Montecillo, MX. Universidad Autónoma Chapingo. $32 \mathrm{p}$

Faostat (FAO Statistical Database). (2012). Production Statistics. Consultado 20 sep. $2014 . \quad$ Disponible en: http://faostat.fao.org/site/339/default.as $\mathrm{px}$

García, A. (2009). Efecto del fraccionamiento de nitrógeno en la productividad y en la eficiencia agronómica de macronutrientes en maíz. IPNI. Informaciones Agronómicas n ${ }^{\circ}$ 72: 1-5

Giménez, F.; Tomaso, J. (2007). Evaluación de cultivares de cebada cervecera en Balcarce Buenos Aires, AR. INTA. p. 1-4

Henríquez, C., Killorn, R., Bertsch, F., y Sancho, F. (2005). La geostadística en el estudio de la variación espacial de la fertilidad del suelo mediante el uso del interpolador kriging. Agronomía Costarricense, 29(2), 73-81

INIAP (Instituto Nacional Autónomo de Investigaciones Agropecuarias). (2010). Informe de actividades 2009 del Convenio INIAP-CORPOINIAPCERVECERÍA NACIONAL. Quito, EC. $16 \mathrm{p}$

INEC (Instituto Nacional de Estadísticas y Censos). 2010. Sistema agroalimentario de la cebada. Consultado 20 sep. 2014. Disponible en: www.inec.gob.ec 
Lazzari, M.; Landriscini, M.; Cantamutto, M.; Miglierina, A.; Rosell, R. Mockel, F.; Echague, M. (2001). Absorción de nitrógeno por cebada cervecera en dos suelos del sur Bonaerense. Ciencia del Suelo. 19(2):101-102
Salisbury, F.; Ross, C. 2000. Desarrollo de las plantas y fisiología ambiental. Madrid, ES. Paraninfo. p. 544-551 